\title{
Precise Pulmonary Function Evaluation and Management of a Patient With Freeman-Sheldon Syndrome Associated With Recurrent Pneumonia and Chronic Respiratory Insufficiency
}

\author{
Jihyun Park, $\mathrm{MD}^{1,2}$, Seong-Woong Kang, $\mathrm{MD}$, $\mathrm{PhD}^{1,2}$, Won Ah Choi, $\mathrm{MD}, \mathrm{PhD}^{1,2}$, \\ Yewon Lee, $\mathrm{MD}^{1,2}$, Han Eol Cho, $\mathrm{MD}^{1,2}$
}

${ }^{1}$ Department of Rehabilitation Medicine and Rehabilitation Institute of Neuromuscular Disease, Gangnam Severance Hospital, Yonsei University College of Medicine, Seoul;

${ }^{2}$ Pulmonary Rehabilitation Center, Gangnam Severance Hospital, Yonsei University College of Medicine, Seoul, Korea

Freeman-Sheldon syndrome (FSS) is a rare distal arthrogryposis syndrome. There are few reports on the respiratory insufficiency of FSS. Additionally, there is no detailed information on pulmonary functional evaluation. A 17-year-old male patient with FSS developed respiratory failure, leading him to be admitted to hospital several times for evaluation and treatment. Of those times he was admitted, two were due to pneumonia. His pulmonary functions were indicative of a restrictive lung disease potentially caused by severe scoliosis. After a non-invasive ventilatorwas applied correctly to the patient, pulmonary hypertension was normalized. His pulmonary function has been maintained for 13 years. Since receiving proper respiratory care, which includes assisted coughing methods, the patient has not developed pneumonia. It is important to properly evaluate the pulmonary function of patients who have FSS and scoliosis to eliminate the risk of long-term respiratory complications.

Keywords Freeman-Sheldon syndrome, Respiratory insufficiency, Respiratory function tests

\section{INTRODUCTION}

Freeman-Sheldon syndrome (FSS) is a rare disorder associated with distal arthrogryposis syndrome. It is characterized by multiple congenital contractures in the upper and lower limbs, as well as the facial muscles.

Received August 20, 2019; Revised September 16, 2019; Accepted October 1, 2019

Corresponding author: Han Eol Cho

Department of Rehabilitation Medicine, Gangnam Severance Hospital, 211 Eonju-ro, Gangnam-gu, Seoul 06273, Korea. Tel: +82-2-2019-3496, Fax: +82-2-2019-3490, E-mail: wtzephyr@yuhs.ac

ORCID: Jihyun Park (https://orcid.org/0000-0002-2172-1072); Seong-Woong Kang (https://orcid.org/0000-0002-7279-3893); Won-Ah Choi (https:// orcid.org/0000-0003-0403-8869); Yewon Lee (https://orcid.org/0000-0002-2001-3824); Han Eol Cho (https://orcid.org/0000-0001-5625-3013).

(c) This is an open-access article distributed under the terms of the Creative Commons Attribution Non-Commercial License (http://creativecommons.org/ licenses/by-nc/4.0) which permits unrestricted noncommercial use, distribution, and reproduction in any medium, provided the original work is properly cited. Copyright ( 2020 by Korean Academy of Rehabilitation Medicine 
Patients with FSS have distinctive facial features such as small oral orifices, prominent nasolabial folds, sunken eyes, high narrow palate, and an H-shaped dimpling of the chin. Mutations in embryonic myosin heavy chain (MYH3) causes FSS [1].

There are several reports regarding the correlation of clinically appreciated features or phenotype and genotype of FSS and its relationship to anesthesia difficulties. However, there are only a few reports of respiratory insufficiency in FSS patients and no detailed information on respiratory functional evaluations [2].

We report a case of a patient with FSS who developed chronic respiratory insufficiency, managed for 13 years. The patient has had a significant improvement in their quality of life from the respiratory care administered.

This study was approved by the Institutional Review Board of the Gangnam Severance Hospital (No. 3-20190232). Written informed consent was obtained.

\section{CASE REPORT}

A 17-year-old male patients was admitted to the pediatric department of Gangnam Severance Hospital due toirritability, lethargy, and dyspnea aggravation on February
4, 1997. He had exhibited symptoms such as sweating, dyspnea on exertion, and generalized edema since December 1996.

He was born with characteristic features of a person with FSS: club feet, bilateral claw hand deformity, pinched lips, and scoliosis (Fig. 1). Surgery was performed to correct the hand and feet skeletal deformities during childhood.

At the time of admission, pneumonia in the right lower lung field, cardiomegaly, and scoliosis were observed on his chest X-ray. The electrocardiogram showed right axis deviation and right atrial hypertrophy. Complete blood count, electrolyte, creatine kinase (CK) and CK-MB were within the normal range. Arterial blood gas analysis (ABGA) in room air showed low $\mathrm{PaO}_{2}$ and high $\mathrm{PaCO}_{2}$ (Table 1). The treatment for pneumonia with antibiotics and $2 \mathrm{~L} / \mathrm{min}$ of oxygen supply were prescribed. The following day, the patient had an altered mental status, and ABGA showed a $\mathrm{PaCO}_{2}$ of $105.0 \mathrm{mmHg}$, suggestive of $\mathrm{CO}_{2}$ narcosis after the use of oxygen supply. After intubation and ventilator application on asynchronized intermittent mandatory ventilation mode, the patient's $\mathrm{SaO}_{2}$ and $\mathrm{PaCO}_{2}$ became normal (Table 1). Echocardiography showed right heart failure with lowered left ventricular
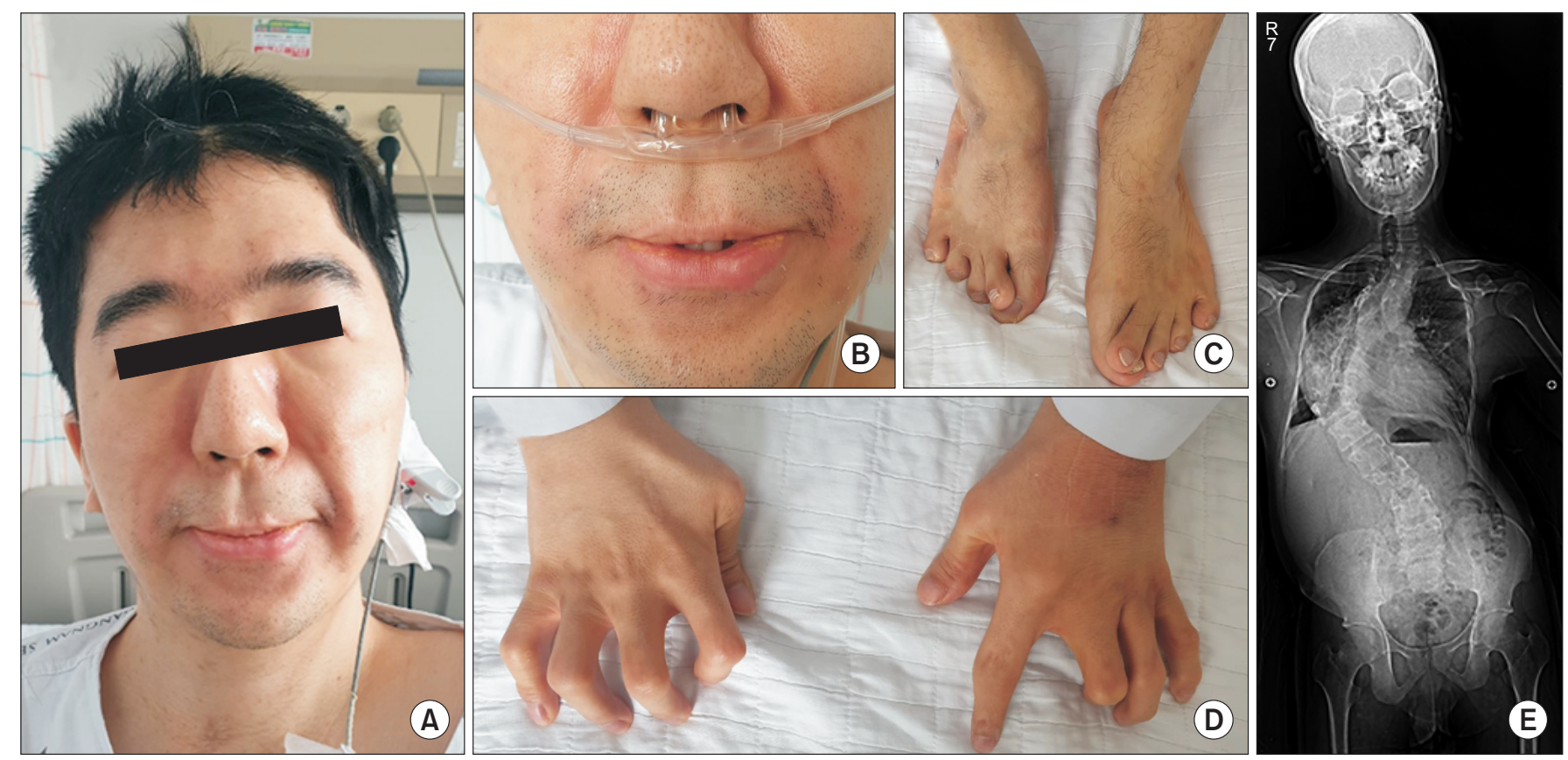

Fig. 1. Characteristic features of Freeman-Sheldon syndrome. (A, B) Face of the patient showing a small mouth with pursed lips, prominent nasolabial folds, a long area between the nose and mouth and sunken eyes. (C,D) Multiple contractures of distal extremities, including camptodactyly. (E) Scoliosis in the patient. 
Table 1. Serial ABGA of the patients

\begin{tabular}{llcccc}
\hline \multicolumn{1}{c}{ Date } & \multicolumn{1}{c}{ Event } & $\mathbf{p H}$ & $\mathbf{P a O}_{\mathbf{2}} \mathbf{( \mathbf { m m H g } )}$ & $\mathbf{P a C O}_{\mathbf{2}} \mathbf{( \mathbf { m m H g } )}$ & $\mathbf{S a O}_{\mathbf{2}}(\mathbf{\%})$ \\
\hline 1997-02-04 & At first admission & 7.352 & 37.5 & 64.1 & 65.3 \\
\hline 1997-02-05 & $\mathrm{O}_{2}$ apply & 7.197 & 78.7 & 105.0 & 93.8 \\
\hline 1997-02-06 & Ventilator apply & 7.579 & 106.8 & 35.4 & 99.3 \\
\hline 1997-03-08 & At discharge without ventilator & 7.325 & 85.5 & 55.4 & 96.6 \\
\hline 2002-01-06 & Admission at the age of 21 & 7.257 & 67.3 & 110.2 & 82.1 \\
\hline 2002-01-20 & At the time of transfer & 7.330 & 87.8 & 78.5 & 96.9 \\
\hline 2002-02-08 & At discharge with ACV mode ventilator & 7.422 & 139.5 & 44.4 & 98.9 \\
\hline 2006-06-26 & Admission at the age of 25 & 7.425 & 146.5 & 4.6 & 99.7 \\
\hline
\end{tabular}

$\mathrm{PaO}_{2}$, partial pressure of arterial oxygen; $\mathrm{PaCO}_{2}$, partial pressure of arterial carbon dioxide; $\mathrm{SaO}_{2}$, arterial oxygen saturation; $\mathrm{ACV}$, assist control ventilation.

Table 2. Echocardiography of the patients

\begin{tabular}{|c|c|c|c|}
\hline Evaluation date & LVEF (\%) & RVSP (mmHg) & RV and RA size \\
\hline 1997-02-12 & 37 & Not checked & Enlarged RV and RA \\
\hline 2002-01-06 & 59 & 41.0 & $\begin{array}{l}\text { Markedly enlarged RV and RA chamber dimensions with } \\
\text { normal sized LV and LA chamber dimension }\end{array}$ \\
\hline 2006-04-12 & 65 & 58.9 & $\begin{array}{l}\text { Markedly enlarged RV and RA chamber dimensions with } \\
\text { normal sized LV and LA chamber dimension }\end{array}$ \\
\hline 2007-08-22 & 62 & 36.4 & Enlarged RV \\
\hline 2008-10-01 & 64 & 18.5 & Decreased RV, normal sized cardiac chamber \\
\hline
\end{tabular}

LVEF, left ventricular ejection fraction; RVSP, right ventricular systolic pressure; RV, right ventricle; RA, right atrium; LV, left ventricle; LA, left atrium.

systolic function (Table 2). Digoxin, Capril, furosemide, and Aldactone were administered due to the possibility of right heart failure. The patient was weaned off the invasive ventilation after pneumonia improved. However, his $\mathrm{PaCO}_{2}$ was still over $50 \mathrm{mmHg}$ at discharge (Table 1).

The patient was repeatedly admitted due to dyspnea and facial swelling in May, August, and September 1997. He underwent intubation and extubation because of $\mathrm{CO}_{2}$ retention whenever he was admitted. In September 1997, he was admitted againwith pneumonia, and treated with a portable mechanical ventilator in the form of bilevel positive airway pressure mode (inspiratory airway pressure $14 \mathrm{cmH}_{2} \mathrm{O}$ and expiratory airway pressure $3 \mathrm{cmH}_{2} \mathrm{O}$ with $3 \mathrm{~L} / \mathrm{min}$ of oxygen). The patient used the ventilator at home as needed when he experienced difficulty to breath for about 0 to 6 hours.

When he was admitted due to dyspnea and facial swelling in January 2002, ABGA showed hypercapnia (Table 1 ), which got better after ventilator application. However, shortly after the ventilator was weaned off, respiratory distress with $\mathrm{PaCO}_{2}$ elevation was observed.
He was transferred to the Pulmonary Rehabilitation Center to receive care for chronic respiratory insufficiency. Anteroposterior radiographic images of the thoracic spine showed right convex scoliosis with a Cobb's angle of $82.4^{\circ}$ with an apex at T10, which can lead to the development of chronic respiratory insufficiency and pulmonary hypertension. His spirometric values were checked. Forced vital capacity (FVC) was checked using a handheld spirometer (Micro Medical Ltd., Rochester, Kent, UK), and peak cough flow (PCF) was measured using a peak-flow meter (Philips Respironics, Guildford, UK). Maximal inspiratory pressure (MIP) and maximal expiratory pressure (MEP) were measured using a mouth pressure meter (Micro Medical Ltd.). He showed decreased FVC, PCF, MIP, and MEP (Table 3).

Since $\mathrm{CO}_{2}$ retention had not been adequately resolved, the ventilation mode was changed from BiPAP to volumetargeted assist control ventilation mode (tidal volume $800 \mathrm{~mL}$, respiratory rate 12 breaths/min with $1 \mathrm{~L} / \mathrm{min}$ of oxygen). The patient was instructed to use a non-invasive ventilator every night and for 2 to 3 hours during the day. 
Jihyun Park, et al.

Table 3. Spirometric values of the patients over time

\begin{tabular}{ccccccccc}
\hline $\begin{array}{c}\text { Evaluation } \\
\text { date }\end{array}$ & $\begin{array}{c}\text { Age at } \\
\text { evaluation }\end{array}$ & FVCsit\% & FVCsup\% & $\begin{array}{c}\text { PCF } \\
\text { (L/min) }\end{array}$ & MIPsit\% & MIPsup\% & MEPsit\% & MEPsup\% \\
\hline $2002-02-04$ & 20.0 & 9.6 & 7.4 & 140 & 32.1 & 26.4 & 42.6 & 39.5 \\
$2006-06-27$ & 24.4 & 18.6 & 12.4 & 160 & 28.2 & 26.5 & 43.1 & 34.2 \\
$2019-07-04$ & 37.4 & 14.3 & 12.8 & 120 & 30.9 & 37.7 & 29.2 & 23.3 \\
\hline
\end{tabular}

FVCsit\%, percent predicted forced vital capacity in sitting position; FVCsup\%, percent predicted forced vital capacity in supine position; PCF, peak cough flow; MIPsit\%, percent predicted maximal inspiratory pressure in sitting position; MIPsup\%, percent predicted maximal inspiratory pressure in supine position; MEPsit\%, percent predicted maximal expiratory pressure in sitting position; MEPsup\%, percent predicted maximal expiratory pressure in supine position.
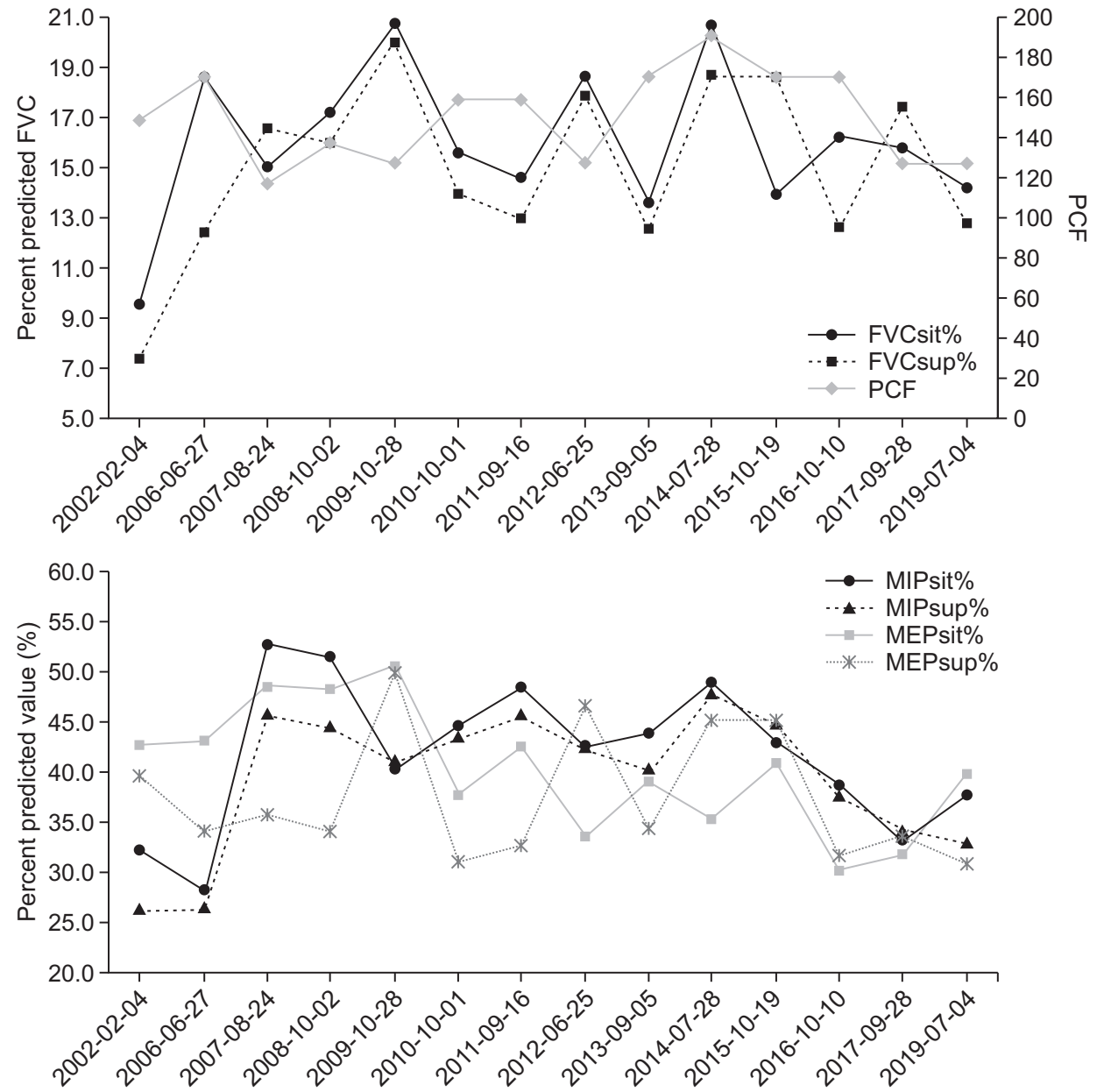

Fig. 2. Serial data of the pulmonary function tests. FVCsit\%, percent predicted forced vital capacity in sitting position; FVCsup\%, percent predicted forced vital capacity in supine position; PCF, peak cough flow; MIPsit\%, percent predicted maximal inspiratory pressure in sitting position; MIPsup\%, percent predicted maximal inspiratory pressure in supine position; MEPsit\%, percent predicted maximal expiratory pressure in sitting position; MEPsup\%, percent predicted maximal expiratory pressure in supine position.
ABGA at discharge showed a decrease in $\mathrm{PaCO}_{2}$, indicating improved respiratory insufficiency (Table 1).

The patient was educated about the importance of conducting regular respiratory function assessments every year to adjust the ventilator. The patient used his ventilator every night but did not visit the pulmonary rehabilitation center until 2006.

He was readmitted to the Pulmonary Rehabilitation
Center and underwent a pulmonary evaluation on June 26, 2006 (Tables 1, 3). Compared to previous results, the patient's predicted percent vital capacity had improved two-fold, and $\mathrm{CO}_{2}$ retention had also improved. However, the findings were much lower than normal, indicating restrictive lung disease. Thereafter, the patient was admitted annually to perform a respiratory function assessment, overnight transcutaneous $\mathrm{CO}_{2}$ monitoring, 
and adjusted ventilator setting. The cardiac function of the patient improved as the ventilation burden on the heart had been reduced with proper usage of the mechanical ventilator. Echocardiography showed moderate pulmonary hypertension (right ventricular systolic pressure [RVSP], $59 \mathrm{mmHg}$ ) in April 2006, and no pulmonary hypertension (RVSP, $18.5 \mathrm{mmHg}$ ) in October 2008. It has been maintained since (Table 2). On November 16, 2017, next-generation sequencing revealed c.2015G $>$ A, a mutation of the MYH3 gene.

The patient's respiratory functions, as evaluated on July 4, 2019, did not show significant deterioration (Table 3, Fig. 2).

\section{DISCUSSION}

There is a possibility of under evaluating respiratory muscle weakness in FSS patients with chronic respiratory insufficiency if only spirometric values are utilized. Patients in previous cases have shown dyspnea, which was misdiagnosed as asthma. After having no response to medication, they were given ventilators $[2,3]$. There were no available reports on the exact spirometric values. In this case, the patient's FVC was checked and noted to be below 20\%, PCF was $160 \mathrm{~L} / \mathrm{min}$, and MEP and MIP were significantly lower than the normal values even after respiratory muscle fatigue and deconditioning were fully resolved. This represents a typical pattern of restrictive lung disease, which might be due to severe scoliosis [4].

Through the current and previous two cases, we assertthat it is important to evaluate the pulmonary function of FSS patients. As scoliosis canprogress quickly and is frequently severe [5], it is important to measure the pulmonary function in patients with FSS. If vital capacity decreases and respiratory insufficiency is observed, noninvasive ventilation should be considered. The patient did not receive adequate respiratory care initially, and this resulted in him developing pulmonary hypertension. After the portable mechanical ventilator was correctly applied and the ventilator settings were adjusted based on pulmonary function observed during an annual follow-up, the patient's pulmonary hypertension was fully resolved, and his pulmonary function improved to some extent. Insufficient breathing volume and secretion problems due to decreased coughing capacity might cause repetitive pneumonia.
The patient's pulmonary function and cardiac function has been maintained for 13 years. There has beena significant improvement in his quality of life as he uses the ventilator only at night and for 2 to 3 hours during the day. He has oxygen via anasal cannula during the day.

The patient was hospitalized twice for pneumonia before receiving proper respiratory care. In 2002, when arespiratory evaluation was first performed, PCF was 150 $\mathrm{L} / \mathrm{min}$. This was insufficient for him to expectorate sputum on his own. The recurrent pneumonia was thought to be caused by decreased cough capacity as reports of bronchopneumonia have been reported previously in FSS patients $[3,6,7]$. There are also reports of postoperative pneumonia and respiratory insufficiency [8].

Since 2006 when he started to receive proper respiratory care, he has not developed pneumonia. His PCF of the assisted coughing technique with maximal insufflation capacity and abdominal thrust increased to $260 \mathrm{~L} / \mathrm{min}$, enough for airway clearance. As scoliosis is prevalent in FSS [5], previous FSS cases with pneumonia might also have failed to induce a sufficient cough to expel mucus from the airways due to weakened respiratory muscle strength. Respiratory muscle weakness may worsen in acutely stressful situations like surgery, leading to respiratory insufficiency or pneumonia in patients who have borderline pulmonary function. Therefore, it is necessary to evaluate pulmonary function including PCF in FSS patients, especially if patients are scheduled for an operation. This measure will not only reduce the incidence of pneumonia and other pulmonary complications with impaired respiratory function [9] but also decrease postoperative risks [10].

It is important to evaluate the pulmonary function and ventilation status in FSS patients because respiratory muscles can be affected by severe scoliosis. Weakened respiratory muscles can lead to frequent respiratory infections and chronic respiratory insufficiency. FSS patients with respiratory muscle weakness may havebetter outcomes if appropriate mechanical ventilation methods and cough training are administered.

\section{CONFLICT OF INTEREST}

No potential conflict of interest relevant to this article was reported. 


\section{AUTHOR CONTRIBUTION}

Conceptualization: Cho HE, Kang SW, Choi WA. Methodology: Cho HE. Formal analysis: Cho HE, Park J, Lee Y. Funding acquisition: Cho HE, Kang SW. Project administration: Cho HE. Visualization: Cho HE, Park J. Writing original draft: Park J, Cho HE. Writing - review, and editing: Cho HE, Kang SW, Choi WA, Lee Y. Approval of final manuscript: all authors.

\section{REFERENCES}

1. Toydemir RM, Rutherford A, Whitby FG, Jorde LB, Carey JC, Bamshad MJ. Mutations in embryonic myosin heavy chain (MYH3) cause Freeman-Sheldon syndrome and Sheldon-Hall syndrome. Nat Genet 2006;38:561-5.

2. Attia A, Suleman M, Al aziz Al Nwasser A. FreemanSheldon syndrome with respiratory failure: a case report. Respir Med CME 2008;1:274-77.

3. Ferrari D, Bettuzzi C, Donzelli O. Freeman-Sheldon syndrome: a case report and review of the literature. Chir Organi Mov 2008;92:127-31.

4. Koumbourlis AC. Scoliosis and the respiratory system.
Paediatr Respir Rev 2006;7:152-60.

5. Stevenson DA, Carey JC, Palumbos J, Rutherford A, Dolcourt J, Bamshad MJ. Clinical characteristics and natural history of Freeman-Sheldon syndrome. Pediatrics 2006;117:754-62.

6. Vanek J, Janda J, Amblerova V, Losan F. Freeman-Sheldon syndrome: a disorder of congenital myopathic origin? J Med Genet 1986;23:231-6.

7. Madi-Jebara S, El-Hajj C, Jawish D, Ayoub E, Kharrat $\mathrm{K}$, Antakly MC. Anesthetic management of a patient with Freeman-Sheldon syndrome: case report. J Clin Anesth 2007;19:460-2.

8. Munro HM, Butler PJ, Washington EJ. FreemanSheldon (whistling face) syndrome: anaesthetic and airway management. Paediatr Anaesth 1997;7:345-8.

9. Miske LJ, Hickey EM, Kolb SM, Weiner DJ, Panitch HB. Use of the mechanical in-exsufflator in pediatric patients with neuromuscular disease and impaired cough. Chest 2004;125:1406-12.

10. Lee JW, Won YH, Kim DH, Choi WA, Bach JR, Kim DJ, et al. Pulmonary rehabilitation to decrease perioperative risks of spinal fusion for patients with neuromuscular scoliosis and low vital capacity. Eur J Phys Rehabil Med 2016;52:28-35. 